\title{
Expression of fascin-1, an actin-bundling protein, in migrating hepatoblasts during rat liver development
}

Yoshihiro Hayashi ${ }^{1 *}$, Katsumi Toda ${ }^{2}$, Toshiji Saibara ${ }^{3}$, Sumika Okamoto ${ }^{1}$, Makoto Osanai $^{1}$, Hideaki Enzan ${ }^{4}$, Gang-Hong Lee ${ }^{1}$

${ }^{1}$ Department of Pathology

${ }^{2}$ Department of Molecular Genetics

${ }^{3}$ Department of Gastroenterology and Hepatology

School of Medicine, Kochi University, Oko-cho, Nankoku, Kochi 783-8505, Japan

${ }^{4}$ Department of Pathology, Chikamori Hospital, Kochi 780-8522, Japan

Key words: fascin, hepatoblast, immunohistochemistry, migration, ultrastructure 
Grant support: This study was supported in part by grant-in-aid for scientific research from Japan Society for the Promotion of Science, and by grant from the Smoking Research Foundation, Japan.

*Corresponding author:

Department of Pathology, School of Medicine, Kochi University, Oko-cho, Nankoku, Kochi 783-8505, Japan

Tel./Fax: +81-88-880-2332; E-mail: jm-hayashiy@kochi-u.ac.jp 


\section{Abstract}

Fascin- 1 is an actin-bundling protein localized at the core actin bundles within microvillar projections and filopodial extensions in migrating cells. It is expressed at a low level in normal epithelial cells, but at a high level in tumor cells, indicating its importance in the invasion and progression of tumor cells. In addition, fascin-1 is expressed in human and murine embryos, especially at high levels in developing nervous tissues. In this study, we immunohistochemically investigated the expression patterns of fascin-1 during the early stages of rat hepatogenesis. A high expression of fascin-1 was detected in the liver bud and hepatoblasts at embryonic day (ED) $10.5,11.5$ and 12.5. The expression fell by ED13.5 and was not detectable at ED14.5. These observations demonstrated that the expression of fascin-1 is correlated with the migration activity of hepatoblasts during the early stages of liver development in rats.

\section{Introduction}


During embryogenesis, the ventral foregut endodermal cells become destined to form the liver. Confined foregut endodermal cells differentiate and proliferate within the epithelium and then begin to migrate into the surrounding septum transversum mesenchyme (Zaret 2000; Duncan 2003). Liver development is based on mutual interaction between the ventral foregut endoderm and two distinct mesenchymal tissues, the precardiac mesoderm and septum transversum mesenchym (LeDouarin 1975; Houssaint 1980; Fukuda-Taira 1981). At around embryonic day 8.0 (ED8.0) in the mouse, a limited region in the ventral foregut endoderm receives inductive signals from these mesodermal tissues (LeDouarin 1975, Gauldi et al. 1996; Jung et al. 1999; Rossi et al. 2001). Likewise, the liver develops from the ventral foregut endoderm at ED10.0 in the rat. Cytokines or transcriptional regulatory factors that control liver specification during early development have been well investigated (Kaestner et al. 1998; Jung et al. 1999; Duncan 2000; Rossi et al. 2001; Zhao and Duncan 2005).

Fascin, an actin-bundling protein, is found in microspikes, membrane ruffle, and stress fibers and is reported to induce membrane protrusions and cell migration (Yamashiro-Matsumura and Matsumura 1998; Adams 2004). 
Furthermore, it supports cellular processes, including microvillar projections and filopodial extensions (Cant and Cooley 1996). Recently, fascin-1 has been shown to be expressed at a high level in mesenchymal and nervous tissues and at a low level or absent in non-pathological epithelial cells (Kureisy et al. 2002; Yamashiro-Matsumura and Matsumura 1998). However, human epithelial tumors, including the esophagus, lung, breast and colon, expressed markedly increased amounts of fascin-1 (Grothey et al. 2000; Jawhari et al. 2003; Goncharuk and Carison 2002; Hashimoto et al. 2005; Pelosi et al. 2003), suggesting that fascin-1 is an important factor for tumor cells to invade and progress. Expression analysis of fascin-1 during embryogeneis has also been performed by in situ hybridization, showing that it is expressed in a variety of tissues including neural tissue, somites, mesenchymal tissue and branchial arches, but not in the liver during mouse embryogenesis (Arcangelis et al. 2004). Most recently, fascin-1 was expressed in the developing nervous system, mesenchymal tissues and gastrointestinal tract, but not in the liver throughout human embryonic and fetal development (Zhang et al. 2008).

The main focus of this study was to examine by 
inmmunohistochemistry whether, and if positive, where and when fascin-1 expression occurs in the rat liver during development. Our results revealed that a high expression of fascin-1 was detectable in the liver bud and hepatoblasts in the septum transversum only at a very early stage of development, ED10.5-12.5. The expression had fallen in most hepatoblasts by ED13.5 and was not detected in hepatoblasts at ED14.5. Thus, these findings provide evidence that fascin-1 is principally expressed in hepatoblasts during the actively migrating period.

\section{Materials and Methods}

Animals and tissues

Pregnant Wistar strain rats were obtained from SLC (Shizuoka, Japan). Animals were kept in a controlled light-dark cycle and fed a standard chow diet and water ad libitum.

Livers of adult rats or embryos were used at various times of gestation, beginning at ED10.5. Embryos were removed from the uteri of mothers 
under ether anesthesia. A portion of the liver was immersed in $20 \%$ formaldehyde for immunohistochemical studies, fixed in $2.5 \%$ glutaraldehyde for transmission electron microscopy, or fixed in $4 \%$ paraformaldehyde (PFA) for immuno-electron microscopy and whole-mount immunohistochemistry. For RT-PCR examination, liver tissues were immersed in RNA-later (Applied Biosystems, CA, USA), and stored at $-20^{\circ} \mathrm{C}$ until use. Samples at all embryonic stages were prepared from at least two different litters.

Immunofluorescent staining

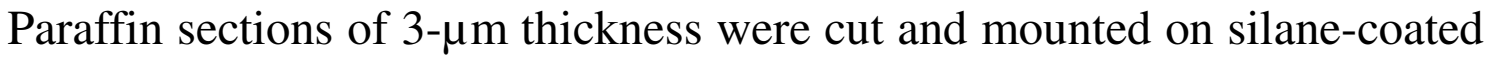
glass slides. The deparaffinized sections were microwaved for $15 \mathrm{~min}$ in $10 \mathrm{mM}$ citric acid $(\mathrm{pH}$ 6.0) for antigen retrieval. The sections were incubated with $10 \%$ normal rabbit serum in $0.01 \mathrm{M}$ phosphate-buffered saline (PBS) for $30 \mathrm{~min}$ at room temperature, and then with monoclonal anti-fascin antibody (55k-2; Dako Carpinteria, CA, USA), which was diluted 1:50 with PBS containing 0.1\% bovine serum albumin (BSA), at 
$4{ }^{\circ} \mathrm{C}$ overnight. After washing with PBS, the sections were incubated with biotinylated rabbit anti-mouse immunogloulin, 1:200 (Dako, Glostrup, Denmark) for $1 \mathrm{hr}$ at room temperature, washed with PBS, and incubated with streptavidin-FITC, 1:200 (Dako, Glostrup, Denmark) for 1hr. After washing, they were counterstained with 4',6-diamidino-2-phenylindole (DAPI), and mounted with fluorescent mounting medium (Dako Carpinteria, CA, USA). As a control, sections were processed without incubation with the anti-fascin antibody. Specimens were photographed with an Olympus digital camera (DP70) mounted on an Olympus fluorescent microscope (BX50).

Whole-mount immunohistochemistry

Each embryo was fixed in 4\% PFA/PBS for $4 \mathrm{hr}$ and immersed in $100 \%$ methanol for $20 \mathrm{~min}$ at $-20^{\circ} \mathrm{C}$. The fixed specimens were then bleached with $2 \%$ hydrogen peroxide in methanol for $1 \mathrm{hr}$ at room temperature to block endogenous peroxidase. The rehydrated specimens were first incubated with $1 \%$ normal rabbit serum in $0.1 \%$ TritonX-100 in PBS 
(PBST) consisting of $1 \%$ skim milk for $1 \mathrm{hr}$ at $4^{\circ} \mathrm{C}$, followed by incubation with anti-fascin antibody in PBST at $4{ }^{\circ} \mathrm{C}$ overnight. After washing 8 times with PBST each for $30 \mathrm{~min}$ at $4^{\circ} \mathrm{C}$, the specimens were incubated with a peroxidase anti-mouse antibody (Simple Stain Nichirei, Tokyo, Japan) overnight at $4^{\circ} \mathrm{C}$. After unbinding the second antibody by washing with PBST, the specimens were incubated in DAB solution for $1 \mathrm{hr}$, and added hydrogen peroxidase to $0.02 \%(\mathrm{v} / \mathrm{v})$. The enzymatic reaction was allowed to proceed until the desired color intensity was reached, and the specimens were rinsed three times in PBST. Specimens were photographed with a digital camera mounted on a microscope.

Electron microscopy

Embryonic tissues fixed in $2.5 \%$ glutaraldehyde in $0.1 \mathrm{MPB}(\mathrm{pH} 7.4)$ for 4 hr at $4{ }^{\circ} \mathrm{C}$ were postfixed in $1 \%$ osmium tetroxide in $\mathrm{PB}$ for $1 \mathrm{hr}$ at $4^{\circ} \mathrm{C}$ followed by dehydration and embedding in epoxy resin. To select optimal areas, semi-thin sections were stained with toluidine blue. Ultra-thin 
sections were stained with uranyl acetate and lead citrate and examined with a JEM 100S electron microscope (JEOL, Tokyo, Japan).

Immuno-electron microscopy

Whole fetuses (ED10.5-12.5) were fixed in 4\% PFA solution for $6 \mathrm{hr}$. Following fixation, the specimens were incubated with $10 \%$ sucrose in PBS overnight. Frozen sections were cut into $10 \mu \mathrm{m}$ slices and washed with PBS. The sections were pre-incubated with $10 \%$ normal rabbit serum for 30 min, and then incubated for $24 \mathrm{hr}$ with anti-fascin antibody at $4{ }^{\circ} \mathrm{C}$. Sections were then incubated with biotinylated rabbit anti-mouse immunogloblin overnight at $4{ }^{\circ} \mathrm{C}$, and finally with avidin-biotin peroxidase complex $(\mathrm{ABC}$ : Vector Laboratories Inc., Burlingame, CA, USA) for $1 \mathrm{hr}$ at room temperature. The peroxidase reaction was developed by incubating sections with $0.02 \% \mathrm{DAB}$ in $0.05 \mathrm{M}$ Tris- $\mathrm{HCl}(\mathrm{pH} 7.6)$ for $1 \mathrm{hr}$ at room temperature and then $0.02 \% \mathrm{DAB}$ in $0.05 \mathrm{M}$ Tris- $\mathrm{HCl}\left(\mathrm{pH}\right.$ 7.6) containing $0.01 \% \mathrm{H}_{2} \mathrm{O}_{2}$ for $10 \mathrm{~min}$. The tissue sections were postfixed with $1 \% \mathrm{OsO}_{4}$ in $0.1 \mathrm{M}$ phosphate buffer ( $\mathrm{pH}$ 7.4) for $1 \mathrm{hr}$, dehydrated in a graded ethanol series, 
and covered with Epon812. Ultrathin sections were observed with a JEOL S100 electron microscope.

RT-PCR protocol

Total RNA was extracted from frozen tissues using TRIzol Reagent (Invitrogen, Carlsbad, CA) according to the manufacturer's instructions. First-strand cDNAs were synthesized with oligo-dt primer and Superscript 3 first-strand synthesis system (Invitrogen, Carlsbad, CA). Each single-stranded cDNA was diluted for subsequent PCR amplification. Standard PCR procedure were carried out in 15 microvolumes of PCR buffer. To detect fascin, the following primers were used: 5'-GGGACTCGGAACTCTTCCTC-3' and 5'-GTAGGCGCCATCATTGAACT-3'. As a quantitative control, glyceraldehydrate-3-phosphate dehydrogenase (GAPDH) was amplified with primer 5'-CTCATGACCACAGTCCATGC-3' and 5'-TTCAGCTCTGGGATGACCTT-3'. PCR conditions were initial denaturation at $94 . \mathrm{C}$ for $7 \mathrm{~min}$, following by 30 cycles of 94 for $30 \mathrm{sec}, 55$ 
for $1 \mathrm{~min}$, and 72 for $1 \mathrm{~min}$, and a final extension step of 72 for $10 \mathrm{~min}$. Each PCR product (15 micro) was visualized by ethidium bromide staining on $6 \%$ acrylamide gel.

\section{Results}

Analysis of fascin-1 protein expression in rat embryos by whole-mount immunohistochemistry

At ED11.5, whole-mount immunohisochemistry showed the expression of fascin-1 in multiple regions. Strong staining was detected in the neural tube, the definitive gut endoderm, forelimb buds and liver bud (Fig. 1A). When examined at ED12.5, the liver, somites, forelimb buds and branchial arches were stained strongly but the heart only weakly (Fig. 1 B). Staining of the transversal sections confirmed that the hepatoblasts were positive for fascin-1 expression (Fig, 1 C, D).

Expression of fascin-1 in rat early liver development 
At ED10.5, the ventral foregut endoderm started to develop and form a liver bud. Fascin-1 was expressed in endodermal cells of the liver bud (Fig. 2A-D). Cell types such as neural epithelial cells and mesenchymal cells of somites were also positive for fascin-1 (Fig. 2A, C). At ED11.5, the expression was detected in migrating hepatoblasts separated from the liver bud and vascular endothelial cells in the septum trasnversum (Fig. 2E, F). Strong immunoreaction was also observed in the cardiac endothelial cells and somites (data not shown). At ED13.5, weak and strong expressions of fascin-1 were observed in the hepatoblasts and sinusoidal endothelial cells, respectively. The expression levels of fascin-1 gradually decreased after this stage in hepatoblasts; however, sinusoidal endothelial cells continued to express fascin-1 at high levels (Fig. 3D). In the liver at ED16.5, numbers of fascin-1-positive hepatoblasts were markedly decreased (Fig. 3E). At ED19.5, high expression was detected in bile duct epithelial cells around the portal vein (data not shown). In the adult liver, both hepatocytes and bile duct epithelial cells were negative for fascin-1, whereas sinusoidal endothelial cells were strongly positive (Fig. 3F). 
Ultrastructural finding of liver development

At an ultrastructural observation at ED10.5, the liver bud contacted the mesencymal cells in the septum transversum through cellular protrusions (Fig. 4A). At ED11.5, migrating hepatoblasts became irregular in shape and had a large number of microvillar projections on the cellular surface (Fig. 4B). Likewise, at ED12.5, cell-cell adhesions between hepatoblasts were loose. Hepatoblasts at this stage had many microvillar projections, especially in the areas of contact between those cells (Fig. 4C). At ED14.5, hemopoietic cells began to appear in the liver. At this stage, hepatoblasts had extremely distorted and irregular shapes and therefore only limited areas were in contact with one another. The connection between hepatoblasts and hemopoietic cells was very close (Fig. 4D). Hepatoblasts had few microvillar projections on the cellular surface except for the lumen of biliary canaliculi. They gradually became rounder and larger after this period.

By immuno-electron microscopy, a strong expression of fascin-1 was 
detected as electron-dense deposits on the cell membrane associated with microvilli (Fig. 5A) in the liver bud at ED10.5. At ED12.5, the products were detected as a dot on the cell membrane (Fig. 5B) and in the cell protrusion (lamellipodia) of hepatoblasts (Fig. 5C).

Analysis of fascin-1 mRNA expression in rat embryos and adult liver

Temporal expression profiles of fascin-1 mRNA were examined by RT-PCR analysis. As shown in Fig. 6, the transcripts were detected throughout all embryonic stages from ED13.5 to 20.5 and the adult liver, albeit with a lesser amount in the adult liver.

\section{Discussion}

In general, fascin is expressed at very low levels in normal epithelial cells (Grothey et al. 2000; Jawhari et al. 2003; Goncharuk et al. 2002; Pelosi et al. 2003); however, its strong expression has been documented in carcinomas of the lung (Pelosi et al. 2003), breast (Grothey et al. 2000), 
skin (Goncharuk et al. 2002), colon (Jawhari et al. 2003), and pancreas (Maitra et al. 2003). Thus, fascins are implicated in vital roles in the migration and invasiveness of tumor cells (Tong et al. 2005).

We analyzed the expression of fascin-1 during the development of rat liver from ED10.5 to ED20.5 as well as the adult by whole-mount and sectioned immunohistochemistry. The results showed that fascin- 1 is highly expressed in the liver bud at ED10.5 and in migrating hepatoblasts at ED12.5 and 13.5 but not at later stages. The expression was confirmed at a transcript level in liver samples. These observations are markedly different from those of the mouse liver, where transcripts of the fascin-1 gene were not detected from ED9.5 to ED14.5 as analyzed by in situ hybridization on whole mounts and sections (Arcangelis et al. 2004). This apparent discrepancy could be attributed to difference in species and/or analytical methods used. Intriguingly, Zhang et al. reported human fetal hepatocytes to be immunohistochemically negative for fascin-1, although hepatic sinusoidal endothelial cells and Kupffer cells were positive (Zhang et al. 2008). In addition, they found positive fascin-1 immunoreactivity in some non-hepatic human embryonal tissues including the nervous system, 
gastrointestinal tract and mesenchyme. Thus, fascin-1 expression in migrating hepatoblasts may be indeed species-specific.

Parallel actin bundles are well known important components for strengthening the actin cytoskeleton and supporting outward extension of the plasma membrane of migrating cells (Hashimoto et al. 2005). Fascin-1 is localized in microvillar projections and filopodial extensions in migrating cells (Adams et al. 1997). The physiological roles of fascin were shown in endothelial cells transfected with a fascin expression vector, where the cells formed an increased number of longer and thicker microvilli on the apical surface and showed the disorganization of cell-cell contacts (Yamashiro et al. 1998). Ultrastructual analyses of rat embryos demonstrated that fascin-1 was detected as a spotted immunodeposition at the cell membrane associated with microvilli of the liver bud at ED10.5 and hepatoblasts at ED12.5; however, hepatoblasts at ED14.5 have few microvillar projections and express fascin-1 at a very low level. These correlations of fascin-1 expression and microvillar projections in hepatoblasts suggest that hepatoblasts increase the expression of actin-bundling factors such as fascin-1 at the stages when they actively 
migrate.

On the other hand, fascin-1 expression has been linked with decreased levels of cell adhesion molecules including E-cadherin and $\beta$-catenin (Tao et al. 1996, Yamashiro et al. 1998). In the present study, membranous fascin-1 immunodeposition was observed at the site of intercellular junction adjacent to microvillar projections. This finding could implicate that fascin-1 also functions as an inhibitor of cellular aggregation, which should facilitate hepatoblastic movement during rat embryogenesis.

In conclusion, we have found that fascin-1, an actin-bundling protein, is expressed in migrating hepatoblasts during early hepatogenesis, but not in their later stages; therefore, these results may imply that fascin-1 plays a significant role in rat early liver development.

\section{References}

Adams JC (2004) Role of fascin in cell adherence and motility. Current Opinion in Cell Biology 16: $590-596$

Arcangelis AD, Georges-Labouesse E, Adams JC (2004) Expression of fascin-1, the encording 
the actin-bundling protein fascin-1, during mouse embryogenesis. Gene Expression pattern 4:637-643

Cant K, Cooley L (1996) Single amino acid mutations in Drosophila fascin disrupt actin bundling function in vivo. Genetics 143:249-58

Chakravarti R, Sapountzi V, Adams JC (2005) Functional role of syndecan-1 cytoplasmic V region in lamellipodial spreading, actin bundling, and cell migration. Mol Biol Cell $16: 3678-91$

Duncan SA (2000) Transcriptional regulation of the liver development. Dev Dyn 219:131-142

Duncan SA (2003) Mechanisms controlling early development of the liver. Mech Dev $120: 19-33$

Fukuda-Taira S (1981) Hepatic induction in the avian embryo : specificity of reactive endoderm and inductive mesoderm. J Embryol Exp Morphol 63:111-125

Gualdi R, Bossard P, Zheng M, Hamada Y, Coleman JR, Zaret KS (1996) Hepatic specification of gut endoderm in vitro:cell signaling and transcriptional control. Genes Dev $10: 1670-1682$

Grothey A, Hashizume R, Sahin AA, McCrea PD (2000) Fascin, an actin-bundling protein associated with cell motility, is upregulated in hormone receptor negative breast cancer. Br J Cancer 83:870-873 
Goncharuk VN, Ross JS, Carison JA (2002) Actin-binding protein fascin expression in skin neoplasia. J Cutan Pathol 29:430-438

Hashimoto Y, Ito T, Inoue H, Okumura T, Tanaka E, Tsunoda S, Higashiyama M, Watanabe G, Imamura M, Shimada Y (2005) Prognostic significance of fascin overexpression in human esophageal squamous cell carcinoma. Clin Cancer Res 11:2597-605

Hashimoto Y, Skacel M, Adams JC (2005) Role of fascin in human carcinoma motility and signaling: prospects for a novel biomarker? Int J Biochem Cell Biol 37:1787-1804

Houssaint E (1980) Differentiation of the mouse hepatic primordium. I. An analysis of tissue interactions in hepatocyte differentiation. Cell Differ 9:269-79.

Jawari AU, Buda A, Jenkins M, Shehzad K, Sarraf C, Noda M, Farthing MJ, Pignatelli M, Adams JC (2003) Fascin, an actin-bundling protein, modulates colonic epithelial cell invasiveness and differentiation in vitro. Am J Pathol 162:69-80

Jung J, Zheng M, Goldfarb M, Zaret KS (1999) Initiation of mammalian liver development from endoderm by fibroblast growth factors.

Science 284:1998-2003.

Kaestner KH, Hiemisch H, Schutz G (1998) Targets disruption of the gene encording hepatocyte nuclear factor 3gamma results in reduction transcription of hepatocyte-specific gene. Mol Cell Biol 18:4245-4251 
Kureishy N, Sapountzi V, Prag S, Anikumar N, Adams JC (2002) Fascin, and their roles in cell structure and function. Bioassays 24:350-361

LeDouarin NM (1975) An experimental analysis of liver development. Med Biol 53:427-455

Maitra A, Adsay NV, Argani P, Iacobuzio-Donahue C, De Marzo A, Cameron JL, Yeo CJ, Hruban RH (2003).Multicomponent analysis of the pancreatic adenocarcinoma progression model using a pancreatic intraepithelial neoplasia tissue microarray. Mod Pathol 16:902-912.

Pelosi G, Pastorino F, Maissoneuve P, Fraggetta F, Iannucci A et al (2003) Independent prognostic value of fascin immunoreactivity in stage 1 nonsmall cell lung cancer. $\mathrm{Br} \mathrm{J}$ Cancer 88:537-547

Rossi JM, Dunn NR, Hogan BLM, Zaret KS (1998) Distinct mesodermal signals, including BMPs from the septum transversum mesenchyme, are required in combination for hepatogenesis from the endoderm. Genes Dev 15:1998-2009

Tao YS, Edwards RA, Tubb B, Wang S, Bryan J, McCrea PD (1996) beta-Catenin associates with the actin-bundling protein fascin in a noncadherin complex. J Cell Biol 134:1271-81

Tong GX, Yee H, Chiriboga L, Hernandez O, Waisman J (2005).Fascin-1 expression in papillary and invasive urothelial carcinomas of the urinary bladder. Hum Pathol 36:741-746

Yamashiro S, Yamakita Y, Ono S, Matsumura F (1998) Fascin, an actibundling protein, induces 
membrane protrusion and increases cell motility of epithelial cells. Mol Biol Cell 9:993-1006

Zhang FR, Tao LH, Shen ZY, Lv Z, Xu LY, Li EM (2008) Fascin expression in human embryonic, fetal, and normal adult tissue. J Histochem Cytochem 56:193-9

Zhao R, Duncan SA(2005) Embryonic development of the liver.. Hepatology 41:956-67 


\section{Fig. 1}

Whole-mount immunohistochemistry for fascin. (a) At ED11.5, high expression of fascin was detected in the neural tube (nt), the definitive gut endoderm, and liver bud (arrow). Paraffin section of A, migrated hepatoblasts, were positive for fascin (b). At ED12.5, high expression was observed in the liver (arrow), somites (s), forelimb buds (fb) and branchial arches (ba) (c). Expression of fascin was detected in hepatoblasts in transversal paraffin sections (d). st: septum transversum. Bar $=50$ micro

\section{Fig. 2}

Expression of fascin during early liver development. (a) Sagittal section of an ED10.5. Liver bud (lb), somites (s) were expressed for fascin. (b) B shows high-resolution images of boxed area in A. (c). Transversal section of an ED10.5 embryo. Liver bud adjacent to the septum transversum (st) was strongly positive for fascin. (d) Boxed areas are enlarged in C. (e,f) At ED11.5, hepatoblasts migrating from the liver bud were strongly positive. Bar $=200$ micro (a.c.e). Bar $=50$ micro $(b, d, f)$ 


\section{Fig. 3}

Early liver development. (a,b) Hematoxylin and eosin staining at ED12.5, 14.5. At. ED12.5, cell-cell adhesion of hepatoblasts was loose. In ED14.5 liver, hemopoietic cells showed a marked increase in number within the parenchyma. The contacts of hepatoblasts and hemopoietic cells were very close. (c) At 12.5, high expression of fascin was detected in hepatoblasts and immature sinusoidal endothelial cells (se). (d) At ED14.5, fascin was expressed at very low levels in hepatoblasts. (e) At 16.5, hepatocytes was negative for fascin, whereas sinusoidal endothelial cells showed high staining. (f) Adult liver, mature sinusoidal endothelial cells were strongly positive. Bar $=50$ micro

\section{Fig. 4}

Electron microscopic observation. (a) At ED10.5 embryonic liver. Liver bud (lb) contacted with mesencymal cells (mc) in the septum transversum (st) through cellular protrusions. (b) At ED11.5, migrating hepatoblasts (hb) became irregular in shape and had a large number of microvillar projections at the cellular surface. (c) At ED12.5, cell-cell adhesions 
between hepatoblasts were loose. Hepatoblasts at this stage had many microvillar projections, especially in areas of contact between hepatoblasts. (d) At ED14.5, hemopoietic cells began to appear in the liver. At this stage, hepatoblasts (hb) had extremely distorted and irregular shapes and therefore only limited areas were in contact with other hepatoblasts. The connection between hepatoblasts and hemopoietic cells was very close.

Bar $=2$ micro

\section{Fig. 5}

Immuno-electron micrograph of fascin (b-d). (a) ED10.5 embryo, high expression of fascin was detected in the liver bud (lb). (b) Boxed areas in (a) were observed. Liver bud lumen had immunoreactions in sparse microvilli and cell membrane (arrows). (c.d) At ED11.5 embryo. (c) Strong immunoreactivity was found on the plasma membrane (arrows) and in the cytoplasm of hepatoblasts. (d) Intense fascin immunodeposition was detected in the lamellipodia-like cell protrusion (arrow) of hepatoblasts. Bar $=2$ micro 


\section{Fig. 6}

Expression level of fascin mRNA during rat hepatogenesis. Total RNAs isolated from embryonic and adult liver were reverse-transcribed, and the cDNA was amplified by PCR using rat fascin and GADPH primers. Fascin mRNA of liver from ED13.5 (lane 1), 14.5 (lane 2), 16.5 (lane 3), 18.5 (lane 4), 20.5 (lane 5), and adult livers (lane 6). Amplification of fascin demonstrated that all samples had similar levels, except for adult liver. 


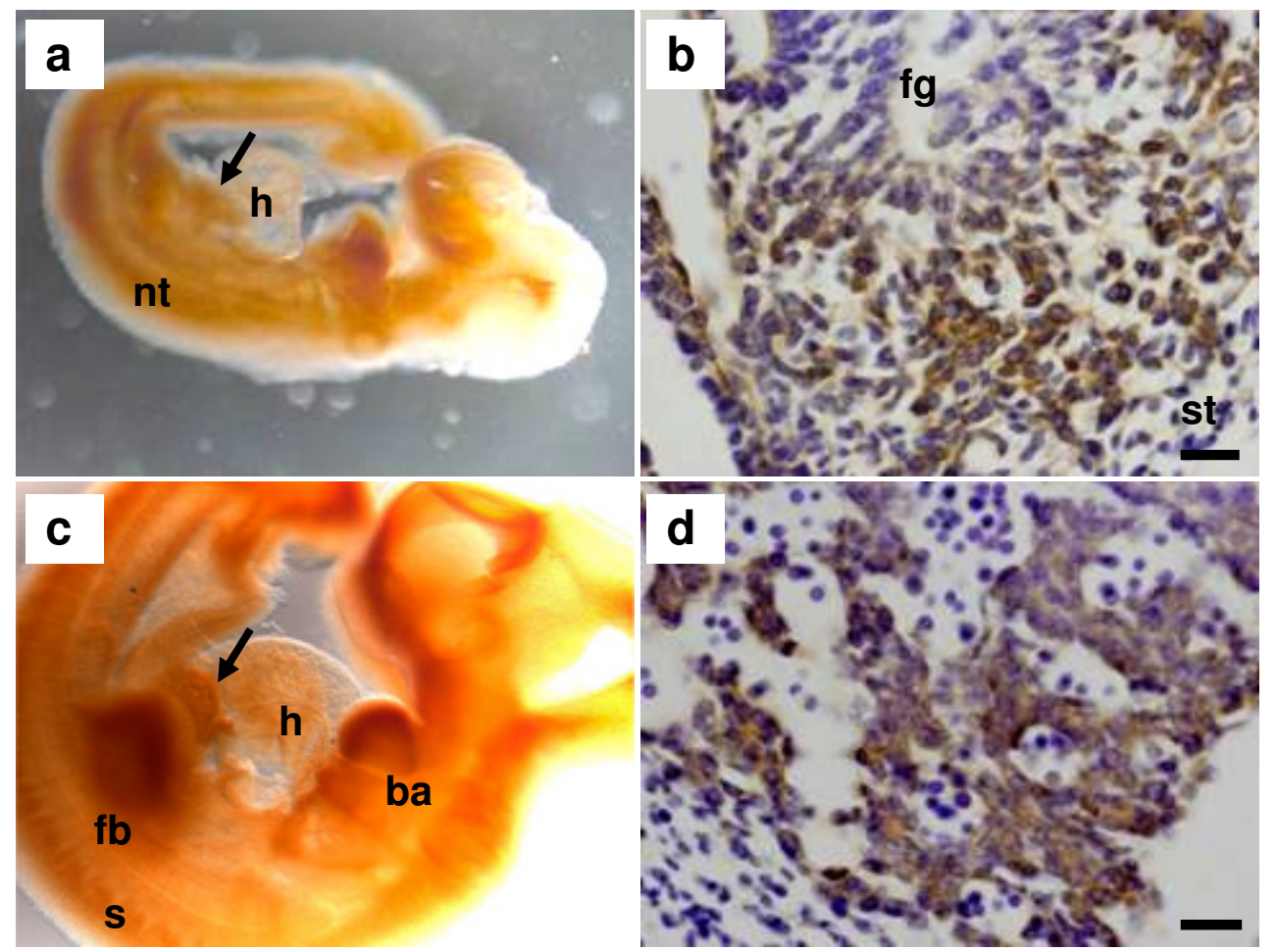

Fig. 1 


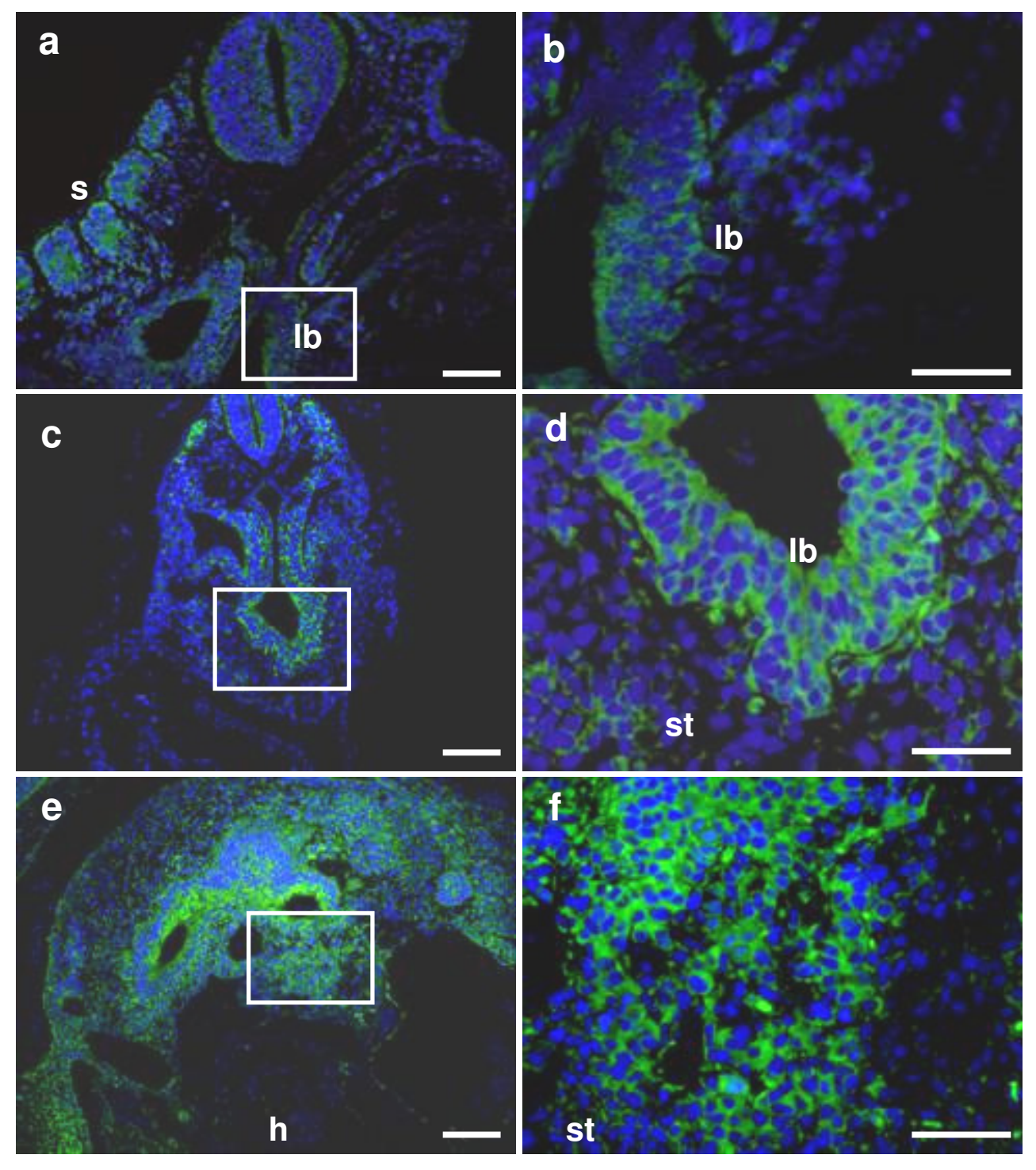

Fig. 2 


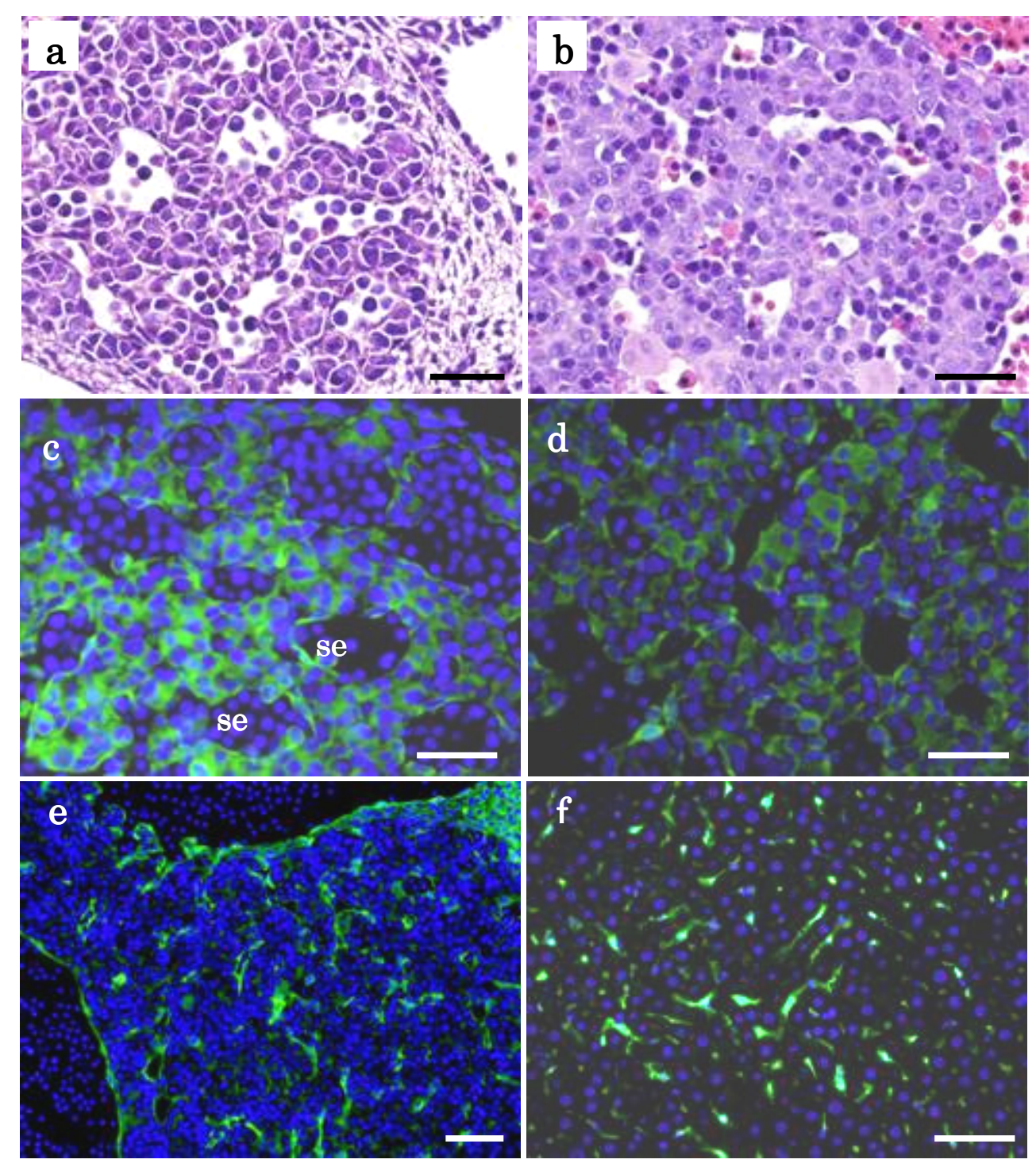

Fig. 3 


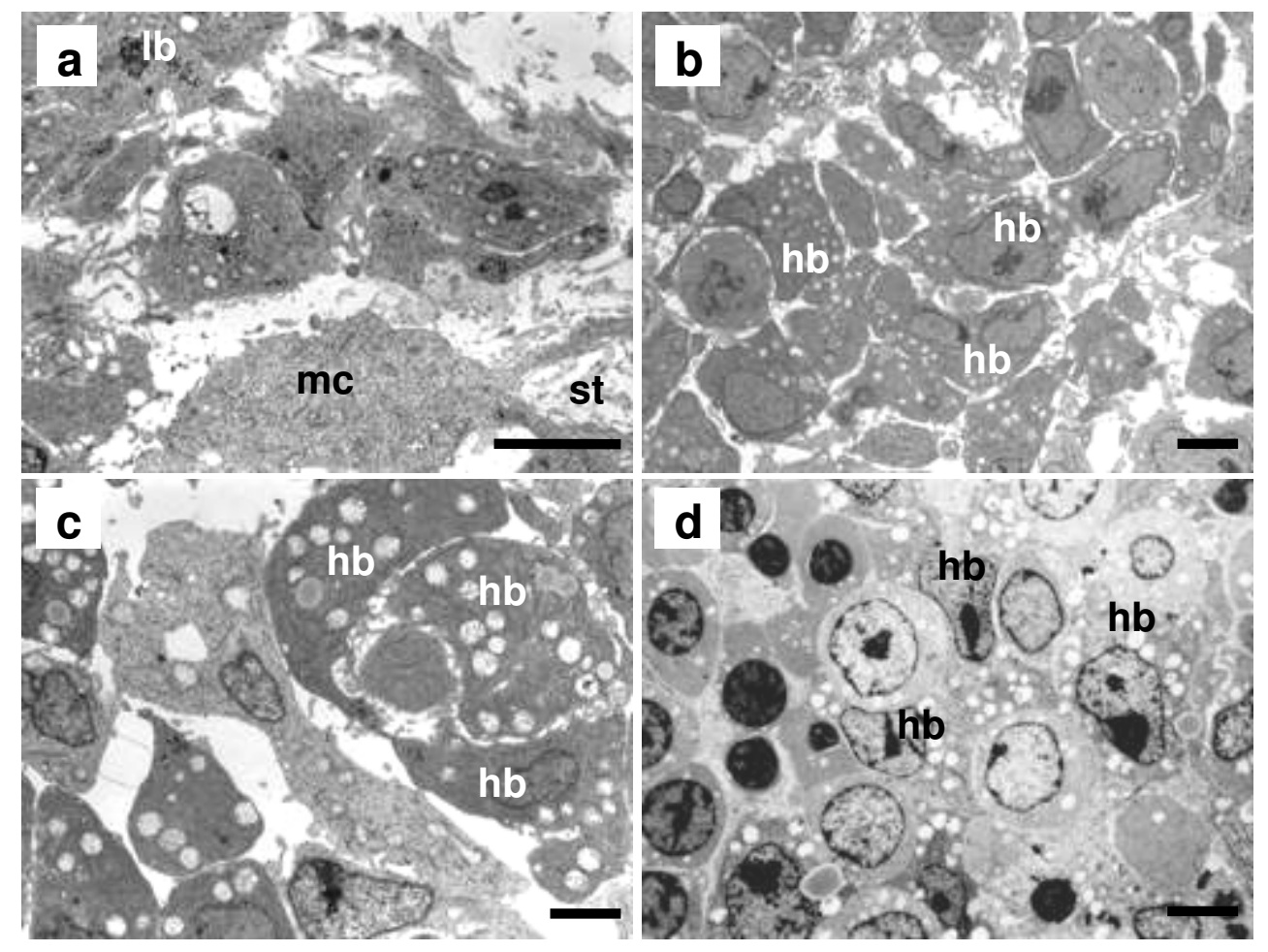

Fig .4 


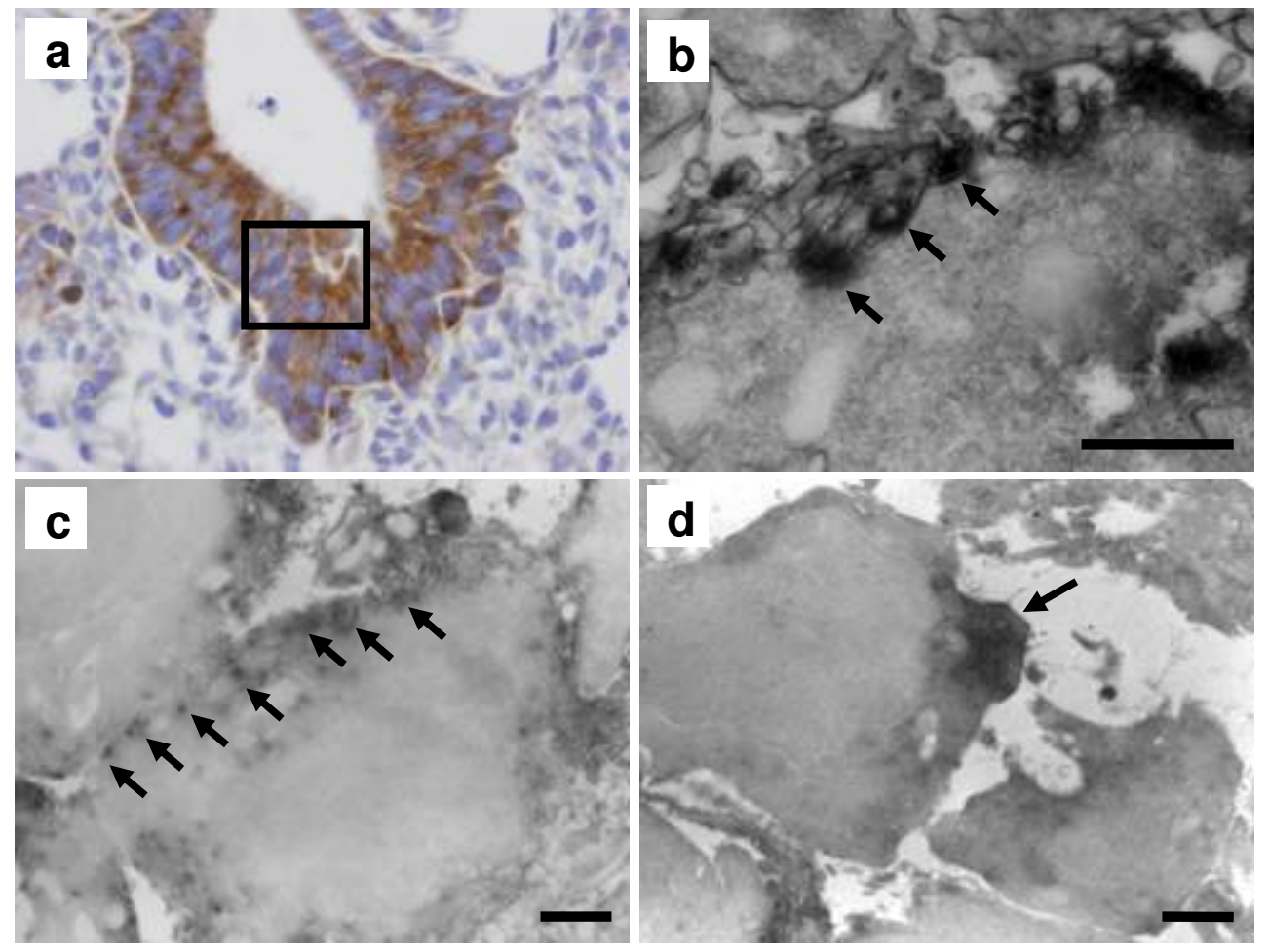

Fig.5 


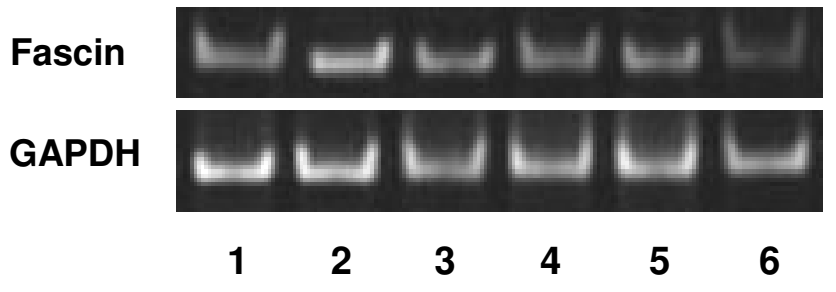

Fig.6 